Commun. Fac. Sci. Univ. Ank. Ser. A1 Math. Stat.

Volume 69, Number 1, Pages 97-111(2020)

DOI: $10.31801 /$ cfsuasmas. 561632

ISSN 1303-5991 E-ISSN 2618-6470

http://communications.science.ankara.edu.tr/index.php?series=A1

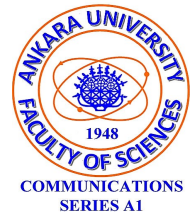

\title{
COMPACTNESS AND STABILITY IN DIFRAMES
}

\author{
ESRA KORKMAZ AND RIZA ERTÜRK
}

\begin{abstract}
The concept of diframe was introduced as a generalization of ditopological texture spaces. The purpose of this paper is to present the results of a study on the concepts of compactness and stability in the setting of diframes. Further, the bitopological concepts of locally compactness and locally stability are extended to diframes.
\end{abstract}

\section{INTRODUCTION}

The theory of bitopological spaces is based on the notion of open sets, and the closed sets can be obtained easily by using the set complementation. As distinct from bitopologies, a ditopological texture space is defined on a suitable subfamily of subsets, which is not necessarily complemented. It can be considered as a structure in which the open and closed sets play an equal role. Diframes were defined in [1] as a generalization of ditopological texture spaces. Briefly, it is a 3 -tuple $L=$ $\left(L_{e}, L_{f r}, L_{c f}\right)$, where $L_{e}$ is both a frame and a coframe, $L_{f r} \subseteq L_{e}$ is a subset closed under arbitrary joins and finite meets and $L_{c f} \subseteq L_{e}$ is a subset closed under arbitrary meets and finite joins. As is well known, point-free topology has a wide range of applications, including logic, topos theory and theoretical computer science. The motivation behind the notion of diframe is to provide a point-free perspective on the theory of ditopological texture spaces. We obtained a larger family of lattices by weakening the property of complete distributivity. This paper is self-contained but may also be considered as a continuation of the article [2], in which we developed the diframe versions of the separation axioms and relations between these axioms. In this study, we are interested in the notions of compactness, stability, local compactness, local stability and their duals in diframes.

The present paper is divided into 5 sections. In Section 2, we present some necessary preliminaries including the concept of compactness in ditopological texture spaces and the separation axioms in diframes. Section 3 is devoted to the study

Received by the editors: May 08, 2019; Accepted: August 20, 2019.

2010 Mathematics Subject Classification. Primary 54A05,54D30; Secondary 06D22.

Key words and phrases. Diframe, compact, stable, locally compact, locally stable.

(C) 2020 Ankara University Communications Faculty of Sciences University of Ankara-Series A1 Mathematics and Statistics 
of compactness and stability in diframes. The questions of whether these properties are hereditary, and whether they are preserved by any reasonable kind of homomorphisms are discussed. As will be seen in the sequel, stability is a property relating the frame $L_{f r}$ and the coframe $L_{c f}$. Hence we replace compactness by stability to obtain diframe versions of topological results relating separation axioms and compactness. In this section, we also give a generalization of Alexander subbase theorem. In section 4, we define the concepts of locally compactness and locally stability in terms of suitable binary relations. For bitopological versions of these concepts, we refer the reader to the comprehensive paper of Kopperman 3 . As expected, the approach of Kopperman is based on the notion of neighbourhood and hence it is dependent on points. Some of our results are parallel to those in [3] but sometimes we need to impose some extra conditions. Finally, in Section 5, we conclude the paper and discuss our future work.

\section{Preliminaries}

In this section, we briefly recall some definitions and results of ditopological texture spaces, (co)frames and diframes which will be used throughout the paper. We refer the reader to [4, 10] for details concerning lattice and frame theory and 6, 7, 8, for details concerning ditopological texture spaces.

Ditopological Texture Spaces: Let $S$ be a set and $S$ be a subset of the powerset $\mathcal{P}(S)$ with the following properties:

(1) $(S, \subseteq)$ is a complete, completely distributive lattice containing $S$ as a top element and $\emptyset$ as a bottom element.

(2) $S$ is point separating.

(3) Arbitrary meet coincides with intersection and finite joins coincide with union in this lattice.

The pair $(S, S)$ is known as a texture space.

A dichotomous topology, (briefly, ditopology) on a texture $(S, S)$ is a pair $(\tau, \kappa)$ of generally unrelated subsets of $S$ satisfying

( $\left.T_{1}\right) S, \emptyset \in \tau$

$\left(T_{2}\right) G_{1}, G_{2} \in \tau \Rightarrow G_{1} \cap G_{2} \in \tau$,

$\left(T_{3}\right) G_{i} \in \tau, i \in I \Rightarrow \bigvee_{i} G_{i} \in \tau$,

$\left(C T_{1}\right) S, \emptyset \in \kappa$,

$\left(C T_{2}\right) K_{1}, K_{2} \in \kappa \Rightarrow K_{1} \cup K_{2} \in \kappa$,

$\left(C T_{3}\right) K_{i} \in \kappa, i \in I \Rightarrow \bigcap_{i} K_{i} \in \kappa$.

Loosely speaking, a ditopology is a structure in which the open and closed sets play an equal role.

Galois Adjunctions: A pair of monotone functions $f: L \rightarrow M, g: M \rightarrow L$ between partially ordered sets is called Galois adjoint if the following condition is satisfied for all $x \in L$ and $y \in M: f(x) \leq y \Leftrightarrow x \leq g(y)$. This fact is referred to by saying that $f$ is a left adjoint to $g$, or $g$ is a right adjoint to $f$. Our notation 
for the adjoints is that of [10], that is, we will denote this adjunction by $f=g^{*}$ or $g=f_{*}$.

One can show that a suprema (resp., infima) preserving map between complete lattices has a right (resp., left) adjoint.

Let $(f, g)$ be a Galois adjunction.

(1) If $L$ and $M$ are complete lattices, then $f$ preserves finite joins and $g$ preserves finite meets.

(2) $f$ is one-one if and only if $g$ is onto.

(3) If $f$ is onto, then $f g=i d$, and if $f$ is one-one, then $g f=i d$.

Frames and coFrames A frame (resp., a coframe) is a complete lattice with the property that binary meet (resp., join) distributes over arbitrary join (resp., meet) and a frame (resp., a coframe) homomorphism is a function between frames (resp., a coframes) preserving arbitrary joins (resp., meets) and finite meets (resp., joins).

Denote by Frm the category of frames, and by Loc its opposite category. The regular subobjects of objects of Loc, sublocales, have various kinds of characterizations. Here we just recall two of them that we shall exploit in the sequel.

Let $L$ be a frame and let $S \subseteq L$ be a subset closed under arbitrary meets. Then $S$ is called a sublocale of $L$ provided that $(x \rightarrow s) \in S$ for all $s \in S$ and $x \in L$. Similarly, if $M$ is a coframe and $S \subseteq M$ is a subset closed under arbitrary joins then $S$ is called a subcolocale of $M$ if $(s \leftarrow x) \in S$ for all $s \in S$ and $x \in M$. Here, " $\rightarrow$ " and " $\leftarrow$ " denote the Heyting and co- Heyting algebra operation, respectively.

A sublocale can also be represented by a nucleus which is a monotone, idempotent, inflationary map preserving finite meets. Note that these two characterizations of a sublocale are equivalent. According to [4], a sublocale $S$ is said to be flat if it is closed under finite joins, or equivalently, if $v_{S}$ preserves finite joins.

Dually, a conucleus $t: M \rightarrow M$ on a coframe $M$ is a monotone, idempotent map preserving finite joins and satisfying $t(x) \leq x$ for all $x \in M$. One can easily show that for a given subcolocale $S \subseteq M, t_{S}(a)=\bigvee\{s \in S: s \leq a\}$ is a conucleus, and conversely, for every conucleus $t: M \rightarrow M, t(M)$ is a subcolocale.

Diframes: A diframe is a 3 -tuple $L=\left(L_{e}, L_{f r}, L_{c f}\right)$ with the following conditions:

(1) $L_{e}$ is a complete lattice satisfying

$$
x \wedge(\bigvee Y)=\bigvee\{x \wedge y: y \in Y\} \text { and } x \vee(\bigwedge Y)=\bigwedge\{x \vee y: y \in Y\}
$$

for any $x \in L_{e}$ and any subset $Y \subseteq L_{e}$.

(2) $L_{f r} \subseteq L_{e}$ is closed under arbitrary joins and finite meets.

(3) $L_{c f} \subseteq L_{e}$ is closed under arbitrary meets and finite joins.

Notice that $L_{e}$ is both a frame and a coframe, $L_{f r}$ is a frame, and $L_{c f}$ is a coframe.

Example 2.1. Consider the family $\Omega_{\mathrm{reg}}(\mathbb{R})$ of regular open sets of $\left(\mathbb{R}, \tau_{s}\right)$, where $\tau_{s}$ is the usual topology on $\mathbb{R}$. If $L_{f r}=\{(-\infty, a): a \in \mathbb{R}\} \cup\{\emptyset, \mathbb{R}\}$ and $L_{e}=L_{c f}=$ $\Omega_{\mathrm{reg}}(\mathbb{R})$ then $L=\left(L_{e}, L_{f r}, L_{c f}\right)$ is a diframe. 
Let $\varphi: L_{e} \rightarrow M_{e}$ be a map preserving arbitrary join and finite meets and satisfying $\varphi\left[L_{f r}\right] \subseteq M_{f r}$, and let $\psi: L_{e} \rightarrow M_{e}$ be a map preserving arbitrary meets and finite joins and satisfying $\psi\left[L_{c f}\right] \subseteq M_{c f}$. Then the pair $(\varphi, \psi)$ is called a diframe homomorphism.

Diframes and diframe homomorphisms form a category diFrm. The dual category of diFrm is denoted by diLoc, and the objects of diLoc are referred to as dilocales.

By a base of a diframe, we mean a subset $\beta \subseteq L_{f r}$ such that for every $a \in L_{f r}$ there exists a $\beta_{a} \subseteq \beta$ with $a=\bigvee \beta_{a}$. Dually, a cobase is a subset $\beta \subseteq L_{c f}$ such that every $k \in L_{c f}$ can be expressed as a meet of some elements of $\beta$.

A subset $\delta \subseteq L_{f r}$ (resp., $\delta \subseteq L_{c f}$ ) is called a subbase (resp., subcobase) of $L$ if the set of finite meets (resp., joins) of $\delta$ is a base (resp., cobase) of $L$.

A diframe homomorphism $(\varphi, \psi):\left(L_{e}, L_{f r}, L_{c f}\right) \rightarrow\left(M_{e}, M_{f r}, M_{c f}\right)$ is called

(1) onto (resp., one-one) if both $\varphi$ and $\psi$ are onto (resp., one-one),

(2) open (resp., co- open) if $\psi^{*}(a) \in L_{f r}$ (resp., $\varphi_{*}(a) \in L_{f r}$ ) for all $a \in M_{f r}$,

(3) closed (resp., co- closed) if $\psi^{*}(k) \in L_{c f}$ (resp., $\varphi_{*}(k) \in L_{c f}$ ) for all $k \in M_{c f}$.

Let us recall the non-full subcategory hdiFrm of diFrm introduced in 11. The objects of hdiFrm are diframes, and the morphisms are mappings $\varphi:\left(L_{e}, L_{f r}, L_{c f}\right) \rightarrow$ $\left(M_{e}, M_{f r}, M_{c f}\right)$ preserving arbitrary meets and joins, and satisfying the properties $\varphi\left[L_{f r}\right] \subseteq M_{f r}, \varphi\left[L_{c f}\right] \subseteq M_{c f}$.

If $\varphi$ is one-one and onto then the concept of openness (resp., closedness) coincides with the concept of co- opennness (resp., co- closedness). A hdiFrm isomorphism is an open, closed, one-one and onto hdiFrm morphism.

Recall that by a subdilocale of a diframe L, we mean a triple $S=\left(S_{e}, S_{f r}, S_{c f}\right)$ where $S_{e} \subseteq L_{e}$ is both a sublocale and a subcolocale of $L_{e}, S_{f r}=v_{S_{e}}\left(L_{f r}\right) \subseteq S_{e}$ and $S_{c f}=t_{S_{e}}\left(L_{c f}\right) \subseteq S_{e}$.

Note that $S_{e} \subseteq L_{e}$ is obviously a flat sublocale, and hence the nucleus $v_{S_{e}}$ preserves finite joins. Similarly, by defining a co- flat subcolocale as subcolocale closed under finite meets, we obtain that the co- nucleus $t_{S_{e}}$ preserves finite meets.

In a diframe $L=\left(L_{e}, L_{f r}, L_{c f}\right)$, we have the closure and interior of $a \in L_{e}$ given by the formulas $[a]=\bigwedge\left\{c \in L_{c f}: a \leq c\right\}$ and $] a\left[=\bigvee\left\{b \in L_{f r}: b \leq a\right\}\right.$, respectively.

Now, we briefly present the separation axioms in diframes. A comprehensive discussion on their basic properties, characterizations and the implications between them can be found in our previous work [2].

A diframe $L=\left(L_{e}, L_{f r}, L_{c f}\right)$ is called

(1) $T_{0}$ if given $a \in L_{e}$, there exist $c_{i}^{j} \in L_{f r} \cup L_{c f}, i \in I, j \in J$ such that $a=\bigvee_{j \in J} \bigwedge_{i \in I} c_{i}^{j}$,

(2) $c o-T_{0}$ if given $a \in L_{e}$, there exist $c_{i}^{j} \in L_{f r} \cup L_{c f}, i \in I, j \in J$ such that $a=\bigwedge_{j \in J} \bigvee_{i \in I} c_{i}^{j}$,

(3) $R_{0}$ if every element of $L_{f r}$ can be written as a supremum of some elements of $L_{c f}$, 
(4) $c o-R_{0}$ if every element of $L_{c f}$ can be written as a infimum of some elements of $L_{f r}$,

(5) $R_{1}$ if for all $a \in L_{f r}, a=\bigvee_{j \in J} \bigwedge_{i \in I} c_{i}^{j}=\bigvee_{j \in J} \bigwedge_{i \in I}\left[c_{i}^{j}\right]$ where $c_{i}^{j} \in L_{f r}$,

(6) $c o-R_{1}$ if for all $\left.a \in L_{c f}, a=\bigwedge_{j \in J} \bigvee_{i \in I} k_{i}^{j}=\bigwedge_{j \in J} \bigvee_{i \in I}\right] k_{i}^{j}$ [ where $k_{i}^{j} \in L_{c f}$.

Recall the following relations defined on $L_{e}$. Let $D=\left\{k / 2^{n}: k, n \in \mathbb{N}, k=0, \ldots 2^{n}\right\}$ denote the set of dyadic rationals.

(1) $a \prec_{f r} \mathrm{~b}$, if $a, b \in L_{f r}$ and if there exists $c \in L_{c f}$ such that $a \leq c \leq b$.

(2) $f \prec_{c f} k$, if $f, k \in L_{c f}$ and if there exists $a \in L_{f r}$ such that $f \leq a \leq k$.

(3) $a \prec \prec_{f r} b$ if $a, b \in L_{f r}$ and if there exists $a_{q} \in L_{f r}$ with $q \in D$ and satisfying

$$
a_{0}=a, a_{1}=b \text {, and } a_{q} \prec_{f r} a_{r} \text { if } q<r .
$$

(4) $k \prec \prec_{c f} f$ if $k, f \in L_{c f}$ and if there exist $k_{q} \in L_{c f}$ with $q \in D$ and satisfying

$$
k_{0}=k, k_{1}=f \text {, and } k_{q} \prec_{c f} k_{r} \text { if } q<r .
$$

A diframe $L=\left(L_{e}, L_{f r}, L_{c f}\right)$ is called

(1) regular if $a=\bigvee\left\{x \in L_{f r}: x \prec_{f r} a\right\}$ for all $a \in L_{f r}$,

(2) co- regular if $c=\bigwedge\left\{x \in L_{c f}: c \prec_{c f} x\right\}$ for all $c \in L_{c f}$,

(3) completely regular if $a=\bigvee\left\{x \in L_{f r}: x \prec{ }_{f r} a\right\}$ for all $a \in L_{f r}$,

(4) completely co- regular if $c=\bigwedge\left\{x \in L_{c f}: c \prec_{c f} x\right\}$ for all $c \in L_{c f}$,

(5) normal if for any $c \in L_{c f}$ and $a \in L_{f r}$ such that $c \leq a$ there exists $b \in L_{f r}$ with $c \leq b \leq[b] \leq a$.

Finally, we recall the definition of a Urysohn relation given in [9]: A Urysohn relation on a partially ordered set $(L, \leq)$ is a binary relation $\triangleleft$ satisfying

(U1) If $a \triangleleft b$ then $a \leq b$,

(U2) $a \leq b \triangleleft c \leq d$ implies $a \triangleleft d$,

(U3) $a \triangleleft b$ implies the existence of $c \in L$ with $a \triangleleft c \triangleleft b$.

As was shown in [2, a diframe is completely regular if and only if there exists a Urysohn relation $\triangleleft$ on $L_{e}$ with the following conditions:

(1) $a \triangleleft b$ implies $[a] \leq] b[$,

(2) $a=\bigvee\left\{x \in L_{f r}: x \triangleleft a\right\}$ for every $a \in L_{f r}$.

\section{Compactness and Stability in Diframes}

The notion of compactness for bitopological spaces has several versions in the literature. By adopting the definiton of Kopperman [3], Brown and Diker [] generalized the notion of compactness to ditopological texture spaces. It was also studied by Brown and Gohar [7. Here, we extend this concept to a broader setting.

Definition 3.1. Let $L=\left(L_{e}, L_{f r}, L_{c f}\right)$ be a diframe and $a \in L_{e}$. Then a subset $G \subseteq L_{f r}$ is called a cover of $a$ if $a \leq \bigvee G$. A subset $K$ of $L_{c f}$ is said to be a cocover of $a$ if $\bigwedge K \leq a$.

Definition 3.2. Let $L=\left(L_{e}, L_{f r}, L_{c f}\right)$ be a diframe and $a \in L_{e}$. 
(1) $a$ is called compact (resp., Lindelöf) if for every cover $G$ of $a$, there is a finite (resp., countable) $H \subseteq G$ such that $a \leq \bigvee H$.

(2) $a$ is called co- compact (resp., co- Lindelöf) if for every co- cover $K$ of $a$, there is a finite (resp., countable) $F \subseteq K$ such that $\bigwedge F \leq a$.

(3) $L$ is compact if the top element $1 \in L_{e}$ is compact, and it is Lindelöf if $1 \in L_{e}$ is Lindelöf.

(4) $L$ is co- compact if the bottom element $0 \in L_{e}$ is co- compact, and it is called co- Lindelöf if $0 \in L_{e}$ is co- Lindelöf.

Note that for each property $\mathrm{P}, L=\left(L_{e}, L_{f r}, L_{c f}\right)$ is said to be bi- P if it is P and co-P.

Remark 3.3. Obviously, (co-)compact implies (co-)Lindelöf but the reverse implication is not necessarily true. If $X$ is a countable set, $L_{e}=L_{f r}=\mathcal{P}(X)$ and $L_{c f}=\{X, \emptyset\}$ then the diframe $L=\left(L_{e}, L_{f r}, L_{c f}\right)$ is Lindelöf but not compact.

Proposition 3.4. Every subdilocale of a compact (resp., co-compact) diframe is compact (resp., co- compact).

Proof. It is clear since $1_{S_{e}}=1_{L_{e}}$ and $S_{e} \subseteq L_{e}$ is closed under arbitrary suprema.

Lemma 3.5. Let $L=\left(L_{e}, L_{f r}, L_{c f}\right), M=\left(M_{e}, M_{f r}, M_{c f}\right)$ be diframes, and let $(\varphi, \psi): L \rightarrow M$ be a one-one, onto diframe homomorphism. Then the following statements hold:

(1) If $(\varphi, \psi)$ is an open (resp., co- open) homomorphism, then for all $b \in M_{f r}$ there exists $a \in L_{f r}$ such that $\psi(a)=b$ (resp., $\varphi(a)=b$ ).

(2) If $(\varphi, \psi)$ is a closed (resp., co-closed) homomorphism, then for all $k \in M_{c f}$ there exists $f \in L_{c f}$ such that $\psi(f)=k$ (resp., $\left.\varphi(f)=k\right)$.

Proof. (1) Suppose that $(\varphi, \psi): L_{e} \rightarrow M_{e}$ is a one-one, onto, open diframe homomorphism and $b \in M_{f r}$. Since $\psi$ is onto, there is an $a \in L_{e}$ with $\psi(a)=b$. On the other hand, $\psi$ being one-one yields $\psi^{*} \psi=1_{L_{e}}$, and hence $\psi^{*} \psi(a)=a=\psi^{*}(b)$. Since $(\varphi, \psi)$ is open, $a=\psi^{*}(b) \in L_{f r}$.

The remaining assertions can be proved similarly.

Proposition 3.6. Suppose $L=\left(L_{e}, L_{f r}, L_{c f}\right)$ and $M=\left(M_{e}, M_{f r}, M_{c f}\right)$ are diframes and $(\varphi, \psi): L \rightarrow M$ is a one-one, onto diframe homomorphism.

(1) If $(\varphi, \psi): L \rightarrow M$ is co- open then $L$ is compact iff $M$ is compact.

(2) If $(\varphi, \psi): L \rightarrow M$ is closed then $L$ is co-compact iff $M$ is co-compact.

Proof. (1) Let $L$ be a compact diframe and $B \subseteq M_{f r}$ be a cover of $1_{M_{e}}$. By Lemma 3.5. for each $b_{i} \in B$, there is an $a_{i} \in L_{f r}$ with $\varphi\left(a_{i}\right)=b_{i}$. Since $\varphi\left(1_{L_{e}}\right)=$ $1_{M_{e}}=\bigvee_{i \in I} \varphi\left(a_{i}\right)=\varphi\left(\bigvee_{i \in I} a_{i}\right)$ and $\varphi$ is one-one, we have $1_{L_{e}}=\bigvee_{i \in I} a_{i}$. Now, compactness of $L$ gives $k_{1}, \ldots, k_{n} \in I$ such that $1_{L_{e}}=\bigvee_{k=1}^{n} a_{i_{k}}$. Applying the map 
$\varphi$ to both sides of the equation gives

$$
1_{M_{e}}=\varphi\left(1_{L_{e}}\right)=\varphi\left(\bigvee_{k=1}^{n} a_{i_{k}}\right)=\bigvee_{k=1}^{n} \varphi\left(a_{i_{k}}\right)=\bigvee_{k=1}^{n} b_{i_{k}}
$$

and hence $M$ is compact.

Conversely, suppose $M$ is compact and $A=\left\{a_{i}: i \in I\right\} \subseteq L_{f r}$ is a cover of $1_{L_{e}}$, that is, $1_{L_{e}}=\bigvee_{i \in I} a_{i}$. Since $\varphi$ preserves arbitrary joins, we have $\varphi\left(1_{L_{e}}\right)=$ $1_{M_{e}}=\varphi\left(\bigvee_{i \in I} a_{i}\right)=\bigvee_{i \in I} \varphi\left(a_{i}\right)$. Then, by compactness of $M$, there is a finite subset $\left\{a_{i_{k}}: k=1, \ldots, n\right\}$ of $A$ such that

$$
\varphi\left(1_{L_{e}}\right)=1_{M_{e}}=\bigvee_{k=1}^{n} \varphi\left(a_{i_{k}}\right)=\varphi\left(\bigvee_{k=1}^{n} a_{i_{k}}\right) .
$$

Thus, $\varphi$ being one-one implies $1_{L_{e}}=\bigvee_{k=1}^{n} a_{i_{k}}$, and hence $L$ is compact.

We now give a generalization of Alexander subbase theorem, the proof of which runs as the same as the one given in [7].

Theorem 3.7. Let $L=\left(L_{e}, L_{f r}, L_{c f}\right)$ be a diframe and $\delta$ be a subbase (resp., subcobase) of $L$. Then $L$ is compact (resp., co- compact) if and only if for every cover (resp., co- cover) $A \subseteq \delta$ there exists a finite cover (resp., co- cover) $B \subseteq A$.

Proof. We just give the sketch of the proof. As we mentioned before, the idea repeats that of [7, Theorem 2.14].

The implication " $\Rightarrow$ " is clear by definition of compactness. For the reverse implication, assume that $A \subseteq L_{f r}$ is a subset such that no finite subset of $A$ covers 1. We claim that $A$ is not a cover of 1 . Now let $G$ be the collection of all subsets $B \subseteq L_{f r}$ such that $A \subseteq B$, and $B$ has no finite subset covering 1 . Then $(G, \subseteq)$ is a poset and it has a maximal element $H$ by Zorn's Lemma. Moreover, $H$ satisfies the properties given below:

(1) Given any $a \in L_{f r}$ with $a \notin H$, there exists $\left\{a_{i}: 1 \leq i \leq n\right\} \subseteq H$ such that $a \vee\left(\bigvee_{i=1}^{n} a_{i}\right)=1$.

(2) For every subset $\left\{a_{i}: a_{i} \notin H, 1 \leq i \leq n\right\} \subseteq L_{f r}$ we have $\bigwedge_{i=1}^{n} a_{i} \notin H$.

(3) For every subset $C=\left\{a_{i}: 1 \leq i \leq n\right\}$ of $L_{f r}$ and every $b \in H$ with $\bigwedge_{i=1}^{n} a_{i} \leq b$, there exists an $a_{j} \in C$ such that $a_{j} \in H$.

We also know that no finite subset of $\delta \cap H$ covers 1 since $\delta \cap H \subseteq H$. By using the properties (1) - (3), we see that $\bigvee H=\bigvee(\delta \cap H)$. Now, if $H$ is a cover of 1 then $\bigvee(\delta \cap H)=1$, which contradicts with the assumption. Thus $H$, and hence $A$, is not a cover of 1 .

As can be easily seen from the definitions, (co-) compactness is not a property relating $L_{f r}$ and $L_{c f}$. Thus we need the following concepts that relate the frame $L_{f r}$ and the coframe $L_{c f}$.

Definition 3.8. Let $L=\left(L_{e}, L_{f r}, L_{c f}\right)$ be a diframe. Then $L$ is called 
(1) stable if every element of $L_{c f}$ other than 1 is compact,

(2) co- stable if every $0 \neq a \in L_{f r}$ is co- compact,

Example 3.9. Consider the the diframe $L=\left(L_{e}, L_{f r}, L_{c f}\right)$ of Example 2.1.

(1) $L$ is not compact since the cover $\{(-\infty, a+n): n \in \mathbb{N}\}$ of $\mathbb{R}$ does not have a finite subset covering $\mathbb{R}$. Further, $L$ is not co- compact. Indeed, the cocover $\left\{\left(a-\frac{1}{n}, a+\frac{1}{n}\right): n \in \mathbb{N}\right\}$ of $0_{L_{e}}=\emptyset$ proves our claim.

(2) $L$ is not co- stable. Indeed, for any $(-\infty, b) \in L_{f r}$, we have

$$
\bigwedge_{n \in \mathbb{N}}\left(a, b+\frac{1}{n}\right)=\operatorname{int}\left(\bigcap_{n \in \mathbb{N}}\left(a, b+\frac{1}{n}\right)\right)=\operatorname{int}(a, b]=(a, b) \subseteq(-\infty, b)
$$

but there is no finite $F \subseteq\left\{\left(a, b+\frac{1}{n}\right): n \in \mathbb{N}\right\}$ such that $\bigwedge F \subseteq(-\infty, b)$. (Here, "int" denotes the interior operator.) Moreover, one can easily show that $L$ is not stable.

The following example shows that compactness does not imply stability, and vice versa.

Example 3.10. (1) Let $\Omega(\mathbb{R})$ be the open set lattice of countable complement topology on $\mathbb{R}$. If $L_{e}=\mathcal{P}(\mathbb{R}), L_{f r}=\Omega(\mathbb{R})$ and $L_{c f}=\{\emptyset, \mathbb{R}\}$ then $\left(L_{e}, L_{f r}, L_{c f}\right)$ is a stable, non-compact diframe.

(2) Let $\mathbb{I}$ be the unit interval equipped with the usual topology. If $L_{f r}=$ $\Omega(\mathbb{I})$ and $L_{c f}=\left\{\emptyset,\left[0, \frac{1}{2}\right), \mathbb{I}\right\}$, then the diframe $\left(\mathcal{P}(\mathbb{I}), L_{f r}, L_{c f}\right)$ is obviously compact. But it is not stable since the element $\left[0, \frac{1}{2}\right) \in L_{c f}$ is not compact. Indeed, the cover $\left\{\left[0, \frac{1}{2}-\frac{1}{n}\right): n \geq 2, n \in \mathbb{N}\right\}$ proves our claim.

The bitopological version of the next proposition was proved in 3 . In our case, we shall impose a stronger condition on diframe $L^{\prime}$ because of the lack of complete distributivity in diframes. We replace the property of being $R_{0}$ by that of being regular. Here, it is worth reminding the reader that our $R_{0}$ and $R_{1}$ are given, respectively, as pseudo-Hausdorff $(\mathrm{pH})$ and weak symmetry (ws) in [3].

Proposition 3.11. If $L=\left(L_{e}, L_{f r}, L_{c f}\right)$ is a co- $R_{0}$, stable diframe and $L^{\prime}=$ $\left(L_{e}, L_{f r}, L_{c f}{ }^{\prime}\right)$ is regular, then $L_{c f} \subseteq L_{c f}{ }^{\prime}$. Dually, if $L=\left(L_{e}, L_{f r}, L_{c f}\right)$ is $R_{0}$, costable and $L^{\prime}=\left(L_{e}, L_{f r}{ }^{\prime}, L_{c f}\right)$ is co-regular then $L_{f r} \subseteq L_{f r}{ }^{\prime}$.

Proof. Let $k \in L_{c f}$. The case $k=1$ being obvious, we assume $k \neq 1$. Since $\mathrm{L}$ is co- $R_{0}$, there exist $a_{i} \in L_{f r}$ such that $k=\bigwedge_{i \in I} a_{i}$. By regularity of $L^{\prime}$, $a_{i}=\bigvee_{j \in J}\left\{x_{i j} \in L_{f r}: x_{i j} \prec_{f r} a_{i}\right\}$. Moreover, $x_{i j} \prec_{f r} a_{i}$ implies the existence of $k_{i j} \in L_{c f}^{\prime}$ such that $x_{i j} \leq k_{i j} \leq a_{i}$. Now we have $k \leq \bigvee_{j \in J} x_{i j}$ for all $i \in I$, and hence by stability of $\mathrm{L}$, there is a finite $J_{0} \subseteq J$ with $k \leq \bigvee_{j \in J_{0}} x_{i j} \leq \bigvee_{j \in J_{0}} k_{i j}$. Thus,

$$
k \leq \bigwedge_{i \in I} \bigvee_{j \in J_{0}} k_{i j} \leq \bigwedge_{i \in I} a_{i} \leq k
$$

and hence $k=\bigwedge_{i \in I} \bigvee_{j \in J_{0}} k_{i j} \in L_{c f}{ }^{\prime}$. 
The dual statement can be proved in a dual manner.

The property of being $R_{0}$ (resp., co- $R_{0}$ ) is generally not inherited by subdilocales but it is hereditary if the diframe is co- stable (resp., stable):

Proposition 3.12. Every subdilocale of a (co-)stable diframe is (co-)stable.

Proof. Obvious, since the joins in $S$ coincide with the joins in $L$.

The following two propositions establish the connection between (co-)stability and separation axioms.

Proposition 3.13. Every stable regular diframe is normal. Dually, every co-stable co- regular diframe is normal.

Proof. Let $c \leq a$ for some $c \in L_{c f}, a \in L_{f_{r}}$. We assume $a \neq 1$ since the case $a=1$ is trivial. By regularity, $c \leq a=\bigvee_{i \in I}\left\{x_{i} \in L_{f r}: x_{i} \prec_{f r} a\right\}$. Since $1 \neq c \in L_{c f}$ is a compact element by stability of $\mathrm{L}$, we have $c \leq \bigvee_{i=1}^{n}\left\{x_{i} \in L_{f r}: x_{i} \prec_{f r} a\right\}$. If $x_{i} \prec f r a$ there exists a $k_{i} \in L_{c f}$ such that $x_{i} \leq k_{i} \leq a$. Thus, by setting $\left.b=\bigvee_{i=1}^{n}\right] k_{i}$ [ we obtain

$$
c \leq \bigvee_{i=1}^{n} x_{i} \leq b \text { and }[b]=\left[\bigvee_{i=1}^{n}\right] k_{i}[] \leq\left[\bigvee_{i=1}^{n} k_{i}\right] \leq \bigvee_{i=1}^{n} k_{i} \leq a
$$

Proposition 3.14. $\quad$ (1) A $R_{1}$ co- stable diframe is regular.

(2) A co- $R_{1}$ stable diframe is co- regular.

Proof. (1) Suppose that $L$ is a $R_{1}$, co- stable diframe, and take any $a \in L_{f r}$. The case $a=0$ is trivial, so let $a \neq 0$. By $R_{1}, a \in L_{f r}$ can be expressed as $a=\bigvee_{i \in I} \bigwedge_{j \in J} c_{i}^{j}=\bigvee_{i \in I} \bigwedge_{j \in J}\left[c_{i}^{j}\right]$ for $c_{i}^{j} \in L_{f r}$. Since, for all i, $\bigwedge_{j \in J}\left[c_{i}^{j}\right] \leq a$ and $L$ is co- stable, there is a finite subset $J_{0} \subseteq J$ of indices such that $\bigwedge_{j \in J_{0}}\left[c_{i}^{j}\right] \leq a$. Set $x_{i}=\bigwedge_{j \in J_{0}} c_{i}^{j}$ for all $i \in I$. Then $\bigwedge_{j \in J_{0}} c_{i}^{j} \leq \bigwedge_{j \in J_{0}}\left[c_{i}^{j}\right] \leq a$ and $\bigwedge_{j \in J_{0}}\left[c_{i}^{j}\right] \in L_{c f}$, and hence $x_{i} \prec_{f r} a$ for all $i \in I$. Therefore,

$$
\bigvee_{i \in I} \bigwedge_{j \in J_{0}} c_{i}^{j} \leq a \leq \bigvee_{i \in I} \bigwedge_{j \in J} c_{i}^{j} \leq \bigvee_{i \in I} \bigwedge_{j \in J_{0}} c_{i}^{j}
$$

that is, $a=\bigvee_{i \in I}\left\{x_{i} \in L_{f r}: x_{i} \prec_{f r} a\right\}$, which shows that $L$ is regular.

The proof of $(2)$ can be done similarly.

Corollary 3.15. Every $R_{1}$ (resp., co- $R_{1}$ ) bi- stable (i.e., stable and co- stable) diframe is normal.

Proof. $L$ is regular by Proposition 3.14 and hence the statement follows from Proposition 3.13 .

We end this section by discussing the preservation of (co-)stability under certain morphisms. 
Proposition 3.16. Let $(\varphi, \psi):\left(L_{e}, L_{f r}, L_{c f}\right) \rightarrow\left(M_{e}, M_{f r}, M_{c f}\right)$ be an onto, oneone diframe homomorphism.

(1) If $(\varphi, \psi)$ is a co-open, co- closed homomorphism and $L$ is stable then $M$ is stable.

(2) If $(\varphi, \psi)$ is an open, closed homomorphism and $L$ is co-stable then $M$ is co-stable.

Proof. Suppose that $L$ is stable, $1_{M_{e}} \neq k \in M_{c f}$ and $\left\{b_{i}: i \in I\right\} \subseteq M_{f r}$ is a cover of $k$. By Lemma 3.5 there exists $1_{L_{e}} \neq f \in L_{c f}$ with $\varphi(f)=k$ and $a_{i} \in L_{f r}$ with $\varphi\left(a_{i}\right)=b_{i}$ for all $i \in I$. Then we have $\varphi(f) \leq \bigvee_{i \in I} \varphi\left(a_{i}\right)=\varphi\left(\bigvee_{i \in I} a_{i}\right)$, and hence $\varphi_{*} \varphi(f) \leq \varphi_{*} \varphi\left(\bigvee_{i \in I} a_{i}\right)$ since $\varphi_{*}$ is an order preserving map. Now, $\varphi$ being onto implies $\varphi_{*} \varphi(f)=i d$, and hence, by stability of $L$, we have $f \leq \bigvee_{k=1}^{n} a_{i_{k}}$. Thus we obtain

$$
\varphi(f) \leq \varphi\left(\bigvee_{k=1}^{n} a_{i_{k}}\right)=\bigvee_{k=1}^{n} \varphi\left(a_{i_{k}}\right)=\bigvee_{k=1}^{n} b_{i_{k}}
$$

which shows that $M$ is stable.

Proposition 3.17. Let $M$ be a stable (resp., co- stable) diframe and $\varphi: L \rightarrow M$ be a one-one hdiFrm morphism. Then $L$ is a stable (resp., co-stable) diframe.

Proof. Suppose that $M$ is stable. Take any element $1_{L_{e}} \neq f \in L_{c f}$ and any cover $\left\{a_{i} \in L_{f r}: i \in I\right\}$ of $f$. Then $\varphi(f) \leq \varphi\left(\bigvee_{i \in I} a_{i}\right)=\bigvee_{i \in I} \varphi\left(a_{i}\right)$. Since $1_{M_{e}} \neq \varphi(f) \in$ $M_{c f}$ and $\varphi\left(a_{i}\right) \in M_{f r}$ for all $i \in I$, stability of $M$ gives $\varphi(f) \leq \bigvee_{k=1}^{n} \varphi\left(a_{i_{k}}\right)=$ $\varphi\left(\bigvee_{k=1}^{n} a_{i_{k}}\right)$. Thus, applying $\varphi_{*}$ on both sides we obtain $f \leq \bigvee_{k=1}^{n} a_{i_{k}}$.

\section{Locally Compact Diframes}

In this section, we introduce two main concepts, that of locally compactness and locally stability in diframes. As pointed out in the introduction, their bitopological versions use the notion of neighbourhood which is a point-based structure. Hence, we first define the following binary relations on $L_{e}$.

Definition 4.1. Let $L=\left(L_{e}, L_{f r}, L_{c f}\right)$ be a diframe and $x, y \in L_{e}$. Then,

(1) $x \ll_{c} y$ iff there exists a compact $k \in L_{e}$ with $x \leq k \leq y$.

(2) $x \ll_{c c} y$ iff there exists a co- compact $a \in L_{e}$ with $x \leq a \leq y$.

(3) $x \ll_{s} y$ iff there exists a compact $k \in L_{c f}$ with $x \leq k \leq y$.

(4) $x \ll_{c s} y$ iff there exists a co- compact $a \in L_{f r}$ with $x \leq a \leq y$.

Remark 4.2. It is an immediate consequence of the definitions that $x \ll_{s} y$ implies $x \ll_{c} y$ for $x, y \in L_{e}$ and $x \ll_{s} y$ implies $x \prec_{f r} y$ for $x, y \in L_{f r}$. On the other hand, it is obvious that $x \in L_{e}$ is compact (resp., co- compact) iff $x \ll_{c} x$ (resp., $x \ll_{c c} x$ ), and in particular, $L$ is compact (resp., co- compact) iff $1 \ll_{c} 1$ (resp., $\left.0 \ll_{c c} 0\right)$.

Note that the following concepts have no counterparts in the theory of ditopological texture spaces. 
Definition 4.3. A diframe $L$ is called

(1) locally compact if $a=\bigvee\left\{x \in L_{f r}: x \ll_{c} a\right\}$ for all $a \in L_{f r}$,

(2) locally co- compact if $k=\bigwedge\left\{x \in L_{c f}: k \ll_{c c} x\right\}$ for all $k \in L_{c f}$,

(3) locally stable if $a=\bigvee\left\{x \in L_{f r}: x \ll_{s} a\right\}$ for all $a \in L_{f r}$,

(4) locally co- stable if $k=\bigwedge\left\{x \in L_{c f}: k \ll_{c s} x\right\}$ for all $k \in L_{c f}$.

Example 4.4. The diframe in Example 2.1 is neither locally compact nor locally co- compact.

Proposition 4.5. Each subdilocale of a locally (co-) compact diframe is locally (co-) compact.

Proof. Let $S$ be a subdilocale of a locally compact diframe $L$ and let $a \in S_{f_{r}}$. Then, $a=\bigvee\left\{x \in L_{f r}: x \ll_{c} a\right\}$. If $x \ll_{c} a$, then there is a compact $k \in L_{e}$ satisfying $x \leq k \leq a$, and then $v_{S_{e}}(x) \leq v_{S_{e}}(k) \leq v_{S_{e}}(a)=a$ by monotonicity of $v_{S_{e}}$. Thus, it suffices to show that $v_{S_{e}}(k)$ is compact, which yields

$$
a=\bigvee\left\{v_{S_{e}}(x) \in S_{f r}: v_{S_{e}}(x) \ll_{c} a\right\}
$$

and completes the proof.

Let $\left\{b_{i} \in S_{f r}: i \in I\right\}$ be a cover of $v_{S_{e}}(k)$, that is, $k \leq v_{S_{e}}(k) \leq \bigvee_{i \in I} b_{i}$. By compactness of k, we obtain a finite $I_{0} \subseteq I$ with $k \leq \bigvee_{i \in I_{0}} b_{i}$. Hence, applying the nucleus $v_{S_{e}}$ and using the fact that $S_{e}$ is a flat sublocale yield

$$
v_{S_{e}}(k) \leq v_{S_{e}}\left(\bigvee_{i \in I_{0}} b_{i}\right)=\bigvee_{i \in I_{0}} v_{S_{e}}\left(b_{i}\right)=\bigvee_{i \in I_{0}} b_{i}
$$

Thus, $v_{S_{e}}(k)$ is compact.

Proposition 4.6. A (co-)regular, (co-)stable, (co-) compact diframe is locally (co-) stable.

Proof. Let $a \in L_{f r}$. The case $a=1$ is clear by compactness of $L$. So, assume that $a \neq 1$. Then, by regularity, $a \in L_{f r}$ can be writen as $a=\bigvee\left\{x \in L_{f r}: x \prec_{f r} a\right\}$. If $x \prec_{f r} a$ then there exists a $k \in L_{c f}$ with $x \leq k \leq a$. Moreover, $k \neq 1$ since $k \leq a$ and $a \neq 1$. Hence, $k$ is compact since $L$ is a stable diframe. Thus we obtain $x \ll_{s} a$ and

$$
a \leq \bigvee\left\{x \in L_{f r}: x \prec_{f r} a\right\} \leq \bigvee\left\{x \in L_{f r}: x \ll_{s} a\right\} \leq a
$$

which shows that $L$ is locally stable.

The dual proof is analogous.

Proposition 4.7. Let $L$ be a diframe.

(1) $L$ is locally stable iff it is regular and locally compact.

(2) $L$ is locally co- stable iff it is co-regular and locally co- compact. 
Proof. (1) The sufficiency is immediate by Remark 4.2. Thus, we only prove the necessity.

Suppose that $L$ is a regular and locally compact diframe and take an arbitrary $a \in L_{f r}$. Then $a \in L_{f r}$ can be expressed as $a=\bigvee\left\{x \in L_{f r}: x \ll_{c} a\right\}$. Further, if $x \ll_{c} a$ then there exists a compact $k \in L_{e}$ such that $x \leq k \leq a$.

Claim 1: $[k] \leq a$.

By regularity of $L$, we have $k \leq a=\bigvee_{i \in I}\left\{x_{i} \in L_{f r}: x_{i} \prec_{f r} a\right\}$. If $x_{i} \prec_{f r} a$, then there is an $f_{i} \in L_{c f}$ such that $x_{i} \leq f_{i} \leq a$. Hence, there exists a finite $I_{0} \subseteq I$ with $k \leq \bigvee_{i \in I_{0}} x_{i} \leq \bigvee_{i \in I_{0}} f_{i} \leq a$ by compactness of $k$. Thus, we obtain

$$
[k] \leq\left[\bigvee_{i \in I_{0}} f_{i}\right]=\bigvee_{i \in I_{0}}\left[f_{i}\right]=\bigvee_{i \in I_{0}} f_{i} \leq a
$$

Claim 2: $[k] \in L_{c f}$ is a compact element.

Let $\left\{a_{i} \in L_{f r}: i \in I\right\}$ be a cover of $[k]$. By regularity of $L$, each $a_{i}$ can be expressed as $a_{i}=\bigvee_{j \in I}\left\{x_{i j} \in L_{f r}: x_{i j} \prec_{f r} a_{i}\right\}$. If $x_{i j} \prec_{f r} a_{i}$ then there is an $f_{i j} \in$ $L_{c f}$ with $x_{i j} \leq f_{i j} \leq a_{i}$. Moreover, the expression $k \leq \bigvee_{i \in I} a_{i}=\bigvee_{i \in I} \bigvee_{j \in I} x_{i j}$, together with the fact that $k$ is compact, implies the existence of finite subsets $I_{0} \subseteq I$ and $J_{0} \subseteq J$ such that $k \leq \bigvee_{i \in I_{0}} \bigvee_{j \in J_{0}} x_{i j}$. Therefore,

$$
[k] \leq \bigvee_{i \in I_{0}} \bigvee_{j \in J_{0}}\left[x_{i j}\right] \leq \bigvee_{i \in I_{0}} \bigvee_{j \in J_{0}} f_{i j} \leq \bigvee_{i \in I_{0}} a_{i}
$$

and hence $[k]$ is compact.

Now we can conclude that, in a regular diframe, $x \leq k \leq a$ and $k$ being compact imply $x \leq[k] \leq a$ and $[k]$ is compact. Thus,

$$
a=\bigvee\left\{x \in L_{f r}: x \ll_{c} a\right\}=\bigvee\left\{x \in L_{f r}: x \ll_{s} a\right\}
$$

and hence $L$ is locally stable.

Proposition 4.8. Every locally (co-)stable diframe is completely (co-)regular.

Proof. Let $L$ be a locally stable diframe. We claim that the relation

$$
\left.a \triangleleft b \text { if there exists a compact } k \in L_{e} \text { such that }[a] \leq k \leq\right] b[
$$

is a Urysohn relation satisfying the following properties:

(1) $a \triangleleft b$ implies $[a] \leq] b[$,

(2) for every $a \in L_{f r}, a=\bigvee\left\{x \in L_{f r}: x \triangleleft a\right\}$.

$(U 1)$ and $(U 2)$ are obvious by definition of the given relation.

For (U3), let $a \triangleleft b$. Then there exists a compact $k \in L_{e}$ with $\left.[a] \leq k \leq\right] b[$ and further, by locally stability of $L] b,\left[=\bigvee_{i \in I}\left\{c_{i} \in L_{f r}: c_{i} \ll_{s}\right] b[\}\right.$. But then there exists a finite subset $I_{0} \subseteq I$ of indices such that $k \leq \bigvee_{i \in I_{0}} c_{i}$ since $k$ is compact. Moreover, $\left.c_{i} \ll_{s}\right] b\left[\right.$ implies that there exists a compact $k_{i} \in L_{c f}$ with $\left.c_{i} \leq k_{i} \leq\right] b[$. 
Now set $d=\bigvee_{i \in I_{0}}\left[c_{i}\right]$. We have,

$$
\left.[a] \leq k \leq \bigvee_{i \in I_{0}} c_{i} \leq\right] \bigvee_{i \in I_{0}} c_{i}[\leq] \bigvee_{i \in I_{0}}\left[c_{i}\right][=] d[
$$

and hence $a \triangleleft d$. On the other hand,

$$
\left.[d]=\bigvee_{i \in I_{0}}\left[c_{i}\right] \leq \bigvee_{i \in I_{0}} k_{i} \leq\right] b[
$$

and $\bigvee_{i \in I_{0}} k_{i}$ is compact since $k_{i}$ is compact for all $i \in I_{0}$. Hence we have $d \triangleleft b$.

Now it remains to show the properties (1) and (2). The first one is clear by definition. For (2), let $a \in L_{f r}$. Then by locally stability of $L$, it can be written as $a=\bigvee\left\{x \in L_{f r}: x \ll_{s} a\right\}$. If $x \ll_{s} a$ then there is a compact $k \in L_{c f}$ with $x \leq k \leq a$ and hence $[x] \leq k \leq a=] a[$. Thus,

$$
a=\bigvee\left\{x \in L_{f r}: x \ll_{s} a\right\} \leq \bigvee\left\{x \in L_{f r}: x \triangleleft a\right\} \leq a
$$

that is, $a=\bigvee\left\{x \in L_{f r}: x \triangleleft a\right\}$.

Proposition 4.9. Let $L=\left(L_{e}, L_{f r}, L_{c f}\right), M=\left(M_{e}, M_{f r}, M_{c f}\right)$ be diframes and $(\varphi, \psi): L \rightarrow M$ be a one-one, onto diframe homomorphism. Then the following statements hold:

(1) If $(\varphi, \psi)$ is co- open then $L$ is locally compact iff $M$ is locally compact.

(2) If $(\varphi, \psi)$ is closed then $L$ is locally co- compact iff $M$ is locally co- compact.

Proof. (1) Suppose that $L$ is locally compact and take any $b \in M_{f r}$. First, by Lemma 3.5. there is an $a \in L_{f r}$ such that $\varphi(a)=b$ and it can be expressed as $a=\bigvee\left\{x \in L_{f r}: x \ll_{c} a\right\}$ by locally compactness of $L$. If $x \ll_{c} a$ then there exist a compact $k \in L_{e}$ with $x \leq k \leq a$. Then, $\varphi(x) \leq \varphi(k) \leq \varphi(a)=b$ since $\varphi$ preserves order.

Now we claim that $\varphi(k)$ is compact. Let $\left\{b_{i} \in M_{f r}: i \in I\right\}$ be an arbitrary cover of $\varphi(k)$. Then, for every $i \in I$ there exists $a_{i} \in L_{f r}$ such that $\varphi\left(a_{i}\right)=b_{i}$, and hence

$$
\varphi(k) \leq \bigvee_{i \in I} b_{i}=\bigvee_{i \in I} \varphi\left(a_{i}\right)=\varphi\left(\bigvee_{i \in I} a_{i}\right)
$$

Applying the map $\varphi_{*}$ and then using the compactness of $k$, we get $k \leq \bigvee_{k=1}^{n} a_{i_{k}}$. Thus,

$$
\varphi(k) \leq \varphi\left(\bigvee_{k=1}^{n} a_{i_{k}}\right)=\bigvee_{k=1}^{n} \varphi\left(a_{i_{k}}\right)=\bigvee_{k=1}^{n} b_{i_{k}}
$$

and hence $\varphi(k)$ is compact.

Now, using the claim it is easy to see that

$$
b=\varphi(a)=\bigvee\left\{\varphi(x) \in M_{f r}: \varphi(x) \ll_{c} b\right\}
$$

which means that $M$ is locally compact. 
Conversely, assume that $M$ is locally compact. Given any $a \in L_{f r}, \varphi(a) \in M_{f r}$ and hence we have $\varphi(a)=\bigvee\left\{y \in M_{f r}: y \ll_{c} \varphi(a)\right\}$. Moreover, for each $y \in M_{f r}$, there is an $x \in L_{f r}$ such that $\varphi(x)=y$. If $\varphi(x) \ll_{c} \varphi(a)$ then there exists a compact $k \in M_{e}$ with $\varphi(x) \leq k \leq \varphi(a)$. Now we obtain $x \leq \varphi_{*}(k) \leq a$. We can easily show that $\varphi_{*}(k)$ is compact. Thus $a=\bigvee\left\{x \in L_{f r}: x \ll_{c} a\right\}$.

The second one can be proved in a similar manner.

Proposition 4.10. Let $(\varphi, \psi):\left(L_{e}, L_{f r}, L_{c f}\right) \rightarrow\left(M_{e}, M_{f r}, M_{c f}\right)$ be an onto, oneone diframe homomorphism.

(1) If $(\varphi, \psi)$ is a co-open, co- closed diframe homomorphism and $M$ is locally stable then $L$ is locally stable.

(2) If $(\varphi, \psi)$ is an open, closed diframe homomorphism and $M$ is locally costable then $L$ is locally co- stable.

Proof. (2) Assume that $M$ is locally co- stable and take an arbitrary $f \in L_{c f}$. Then we have $\psi(f) \in M_{c f}$ and it can be written as $\psi(f)=\bigwedge\left\{y \in M_{c f}: \psi(f) \ll_{c s} y\right\}$. For all $y$, there is an $x \in L_{c f}$ with $\psi(x)=y$, and if $\psi(f) \ll_{c s} \psi(x)$ then we have a co- compact $b \in M_{f r}$ such that $\psi(f) \leq b \leq \psi(x)$. Moreover, for $b \in M_{f r}$, there is an $a \in L_{f r}$ with $\psi(a)=b$. As in the previous proof, one can see that $a \in L_{f r}$ is co- compact. Thus, we obtain $f=\bigwedge\left\{x \in L_{c f}: f \ll_{c s} x\right\}$, which completes the proof.

Proposition 4.11. The image of a locally stable (resp., locally co- stable) diframe under a one-one, onto, open (resp., closed) hdiFrm morphism is locally stable (resp., locally co- stable).

Proof. This can be proved easily in a similar way used in the proof of the previous propositions.

\section{Conclusion}

In this paper we have introduced the concept of compactness in diframes. Then we have defined stable, locally compact and locally stable diframes and investigated the relations between separation axioms and these properties. As a future work, other topological and bitopological structures such as paracompactness, connectedness and uniformities etc. can be constructed on diframes.

\section{REFERENCES}

[1] Korkmaz E. and Ertürk R., On a new generalization of ditopological texture spaces, Journal of Intelligent 83 Fuzzy Systems, 35, No. 5 (2018) 5529-5539.

[2] Korkmaz E. and Ertürk R., Separation axioms in diframes, European Journal of Pure and Applied Mathematics, 11, No. 3 (2018) 612-627.

[3] Kopperman R., Asymmetry and duality in topology, Topology and its Applications, 66, No. 1 (1995) 1-39.

[4] Johnstone P.T., Stone spaces, Cambridge University Press, 1986. 
[5] Picado J. and Pultr A., Frames and locales. Topology without points, Springer Basel AG, 2012.

[6] Brown, L. M. and Diker M., Ditopological texture spaces and intuitionistic sets, Fuzzy sets and systems, 98 , No. 2 (1998) 217-224.

[7] Brown, L. M. and Gohar M. M., Compactness in ditopological texture spaces, Hacettepe Journal of Mathematics and Statistics, 38, No. 1 (2009) 21-43.

[8] Brown, L. M., Ertürk R., and Dost Ş., Ditopological texture spaces and fuzzy topology. III. Separation axioms, Fuzzy sets and systems, 157, No. 14 (2006) 1886-1912.

[9] Good, C., Kopperman R., and Yıldız F., Interpolating functions, Topology and its Applications, 158, No. 4 (2011) 582-593.

[10] Picado J. and Pultr A., Frames and locales. Topology without points, Springer Basel AG, 2012.

Current address: Esra Korkmaz: Hacettepe University, Department of Mathematics, Ankara, Turkey.

E-mail address: esrakaratas@hacettepe.edu.tr

ORCID Address: http://orcid.org/0000-0002-1065-5612

Current address: Riza Ertürk: Hacettepe University, Department of Mathematics, Ankara, Turkey.

E-mail address: rerturk@hacettepe.edu.tr

ORCID Address: http://orcid.org/0000-0001-7651-6079 